
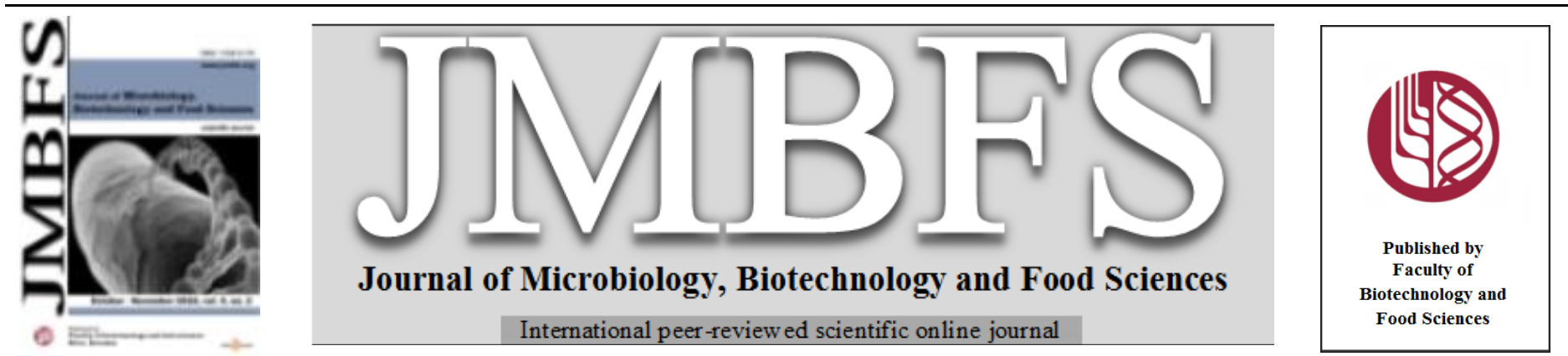

\title{
IMPLICATIONS OF ANTIBIOTIC RESISTANCES PRODUCED BY PHENOTHIAZINES IN Mycobacterium tuberculosis
}

\author{
Address(es): \\ ${ }^{1}$ Department of Botany, Sri Chaitanaya College, Habra, North 24-Parganas, West Bengal, India. \\ ${ }^{2}$ Department of Microbiology, Herbicure Healthcare Bio-Herbal Research Foundation, Boral, Kolkata 700154, India. \\ ${ }^{3}$ Department of Physics, Jadavpur University, Kolkata 700032, India.
}

Sanchayita Debnath ${ }^{1}$, Debalina Sinha Roy ${ }^{2}$, Bidisha Ghosh ${ }^{3}$, Shauroseni Palchoudhuri ${ }^{2}$, Sukhen Das ${ }^{3}$, Sujata G. Dastidar ${ }^{2 *}$

*Corresponding author: jumicrobiol@yahoo.co.in

doi: 10.15414/jmbfs.2016.6.2.781-785

\section{ARTICLE INFO}

Received 30. 10. 2015

Revised 10. 2. 2016

Accepted 21.3.2016

Published 3. 10. 2016

\section{Regular article}

OPEN OACCESS

\begin{abstract}
Several chemotherapeutic agents applied to human beings for past few decades for different ailments, have been found to possess potent antitubercular activity. Two such agents, methdilazine $(\mathrm{Md})$ and promethazine $(\mathrm{Pz})$ were used to select mycobacterial mutants resistant to themselves at different levels and tested to determine if such mutants simultaneously developed cross-resistance to known antitubercular drugs. Mutants were produced by application of a heavy inoculum on Lowenstein-Jensen medium containing Md (or Pz) at concentrations higher than their respective minimum inhibitory concentrations (MICs). These were then tested to find out if such mutants have changed their resistogramme pattern with respect to the test antitubercular agents. Certain first-step Md-mutants became simultaneously resistant to ethambutol and pyrazinamide; while the step-2 Md-mutants revealed further increase in resistance to these agents along with resistance to isoniazid, rifampicin and streptomycin as well. In the study with Pz it was noted that many mutants showed distinctly higher levels of resistance to all the test drugs, particularly to isoniazid, pyrazinamide and streptomycin. The levels of MIC were distinctly high in many mutants. These observations on cross-resistances seem to be best explained on the basis of a reduction in cell-membrane permeability acting in a non-specific manner. The role of such a cross-resistance may possibly be accounting for an overall increase in the MICs of many drugs against several groups of microorganisms including Mycobacterium tuberculosis and other species of Mycobacterium during the last five decades.
\end{abstract}

Keywords: Cross-resistance; Mycobacterium tuberculosis; phenothiazines; mutants; antitubercular agents

\section{INTRODUCTION}

Tuberculosis is a remarkably high risk communicable disease of human beings. The causative organism Mycobacterium tuberculosis is airborne and is often transmitted among people of lower income group suffering from malnutrition and immunological deficiencies. The World Health Organization (WHO, 2013) reported a total of 8.6 million new cases of tuberculosis and 1.3 million deaths due to this infection in 2012. In the treatment regime referred as Directly Observed Treatment Schedule (DOTS) isoniazid, rifampicin, ethambutol and pyrazinamide are administered for two months after presumptive diagnosis. Subsequently isoniazid and rifampicin are continued in the same patients for 4 to 7 months depending on the severity of infection. However, now there are more virulent forms designated as multi-drug resistant strains that are resistant to isoniazid and rifampicin. Apart from this many strains are found to be resistant to isoniazid, rifampicin, streptomycin, any fluoroquinilone plus any of the antitubercular injectables like amikacin/ kanamycin/ capreomycin. Such strains have evolved due to misuse or overuse of the scheduled drugs or failure in continuation of the correct therapy. These strains may also arise if the treatment schedule is allowed to continue for more than 12 months. Simultaneous application of so many drugs creates sufficient pressure for the causative organism to select multi-drug resistant mutants. Such a situation could not have occurred by a simple mode of action since different drugs have different sites of action. The modes of action of anti-tubercular drugs are very varied, although structurally similar drugs usually have the same mutated target.

The occurrence and prevalence of tuberculosis by drug resistant organism initiated systematic search for antimycobacterial agents from various existing pharmacological agents by several groups of researchers in different parts of the world. Such studies revealed that antipsychotic and antihistaminic phenothiazines possess powerful antitubercular action. Most potent among these were thioridazine, methdilazine, trifluoperazine, chlorpromazine and promethazine (Molnar et al., 1977; Kristiansen and Vergemann, 1986; Ratnakar and Murthy, 1993; Chakrabarty et al., 1993; Dutta et al., 2009; Crowle et al., 1992; Amaral et al.,1996; van Ingen et al., 2009; Advani et al., 2012
Kristiansen et al., 2015). These were reported to be simultaneously active against a large number of Gram positive and Gram negative bacteria as well (Kristiansen, 1979; Radhakrishnan et al., 1999; Mazumdar et al., 2001; Dastidar et al., 1995; Dastidar et al., 2004; Dastidar et al., 2013).

Occurrence of cross-resistances among aminoglycosides in Mycobacterium tuberculosis was reported as far back as 1959 by several workers (Torii et al. 1959; Tsukamura, 1959). Koseki \& Okamoto (1963) presented evidences for a significant change in resistance to viomycin caused by development of resistance to capreomycin in Mycobacterium tuberculosis. Tsukamura (1969) while trying to produce drug resistant mutants in the laboratory found that highly kanamycin resistant M.tuberculosis strains were resistant to capreomycin and strains moderately resistant to kanamycin were susceptible to capreomycin. He further observed that experimentally produced capreomycin resistant strains failed to develop resistance to kanamycin. Such a one way cross-resistance relationship was observed among many other aminoglycosides (Tsukamura, 1974). Tsukamura and Mizuno (1975) while trying to determine cross-resistance relationships among aminoglycosides in M.tuberculosis reported that resistances to several antibiotics could be produced by a single mutation to any one of the agents. In 2005 Maus et al attempted to analyze cross-resistance to capreomycin, kanamycin, amikacin and viomycin in M.tuberculosis at molecular level According to these authors mutation of the thyA gene confer capreomycin and viomycin resistance in M.tuberculosis. It is known that in mutations in the $16 \mathrm{~S}$ rRNA gene ( $r r s)$ have been associated with resistance to all these four drugs (Suzuki et al., 1998). Maus et al (2005) reported three rrs mutations in their M.tuberculosis test strains each of which was associated with a particular crossresistance pattern. They opined that when M.tuberculosis strains are exposed to one or two drugs phenotypic and genotypic differences can be seen in the development of antibiotic cross-resistance.

In the present study we have tried to determine the ability of antitubercular phenothiazine compounds methdilazine and promethazine to produce mutants resistant to themselves and also to detect if such mutants develop crossresistances to known antitubercular agents. 


\section{MATERIAL AND METHODS}

\section{Strains}

M.smegmatis 789, M.phlei L1, M. avium 724, M.flavescens 1541, M. gordonae 1324, M.intracellulare 1406, M.tuberculosis $\mathrm{H}_{37} \mathrm{Rv} 102$ and M.tuberculosi $\mathrm{H}_{37} \mathrm{Ra} 16$ were obtained from Dr V.M. Katoch, the then Director of National JALMA Institute for Leprosy and other Mycobacterial Diseases, Agra, India Remaining three strains M.tuberculosis Bajaj 1, J15, and N23 were obtained from Dr A.N. Chakrabarty, Department of Medical Microbiology \& Parasitology, Calcutta University College of Medicine, Kolkata, India. All the organisms were received as live culture slants in Lowenstein-Jensen Medium (LJM) from both the institutions and maintained in the same medium throughout the study.

\section{Media}

All the biological components were obtained from Oxoid (UK). Kirchner's Liquid Medium (KLM) and LJM were prepared as per established protocol (Barrow and Feltham, 2003).The growth was confirmed for Mycobacterium spp. after performing Z-N staining and different biochemical tests, like niacin test, nitrate reduction test and catalase test (Kamerbeek et al., 1997)

\section{Overlay medium}

This was prepared with 7H10 agar base, distilled water and glycerol, distributed in $2 \mathrm{~mL}$ amounts and sterilized by autoclaving.

\section{Inoculation}

The known standard strains M.tuberculosis $\mathrm{H}_{37} \mathrm{Rv} 102$ and $\mathrm{H}_{37} \mathrm{Ra} 16$ along with all the other mycobacteria were grown in KLM, vortexed, diluted and standardized. Mcfarland standard 0.5 (in turbidity standard; the turbidity standard was prepared by adding $0.5 \mathrm{ml}$ of a barium chloride solution to $99.5 \mathrm{ml}$ of $1 \%$ $\mathrm{H}_{2} \mathrm{SO}_{4}$ ) was routinely taken for inoculation of all the strains and their mutants.

\section{Media containing antitubercular drugs and other selecting agents}

The agents were obtained in pure dry powder form from their manufacturers in India and stored at $4^{\circ} \mathrm{C}$. To $2 \mathrm{ml}$ of overlay medium was added, any of the following agents: isoniazid acid hydrazide (INH), rifampicin (Rf), ethambutol $(\mathrm{Eb})$, pyrazinamide $(\mathrm{Pz})$, streptomycin $(\mathrm{Sm})$, methdilazine $(\mathrm{Md})$ and promethazine $(\mathrm{Pz})$. The final concentration $(\mu \mathrm{g} / \mathrm{ml})$ of each of the agents were: $1,2,5,10,25,50,100,200,400,800,1000$ and 2000; these were added to the overlay medium before being allowed to flow over the freshly prepared LJM slants. All such bottles were inoculated for appearance of growth.

\section{Determination of Minimum Inhibitory Concentration (MIC)}

The MIC of the agents for different strains of Mycobacterium and their mutants were determined by inoculating the media as described above and incubating at $37^{\circ} \mathrm{C}$. Appearance of growth was checked every day up to 3 weeks. Adequate strain and media controls were run throughout the tests.

\section{Production and isolation of $\mathrm{Md}$ and $\mathrm{Pz}$ mutants}

This was done by application of a heavy inoculum on LJM containing Md, the amount being higher than the MIC of the organism with respect to the agent. As the mutants developed they were designed as Step 1 mutants and were inoculated on LJM containing still higher amount of Md. The colonies developing on such a medium were designated as Step 2 mutant. In this way higher steps of mutants were selected with both $\mathrm{Md}$ and $\mathrm{Pz}$. The relationship of the mutants with respect to the original wild types was determined with the help of various known and standardized morphological and biochemical parameters as described earlier.

\section{RESULTS AND DISCUSSION}

\section{Sensitivity of bacteria}

The minimum inhibitory concentration (MIC) of INH, Rf, Eb, Py,Sm, Md nd Pz with respect to 20 strains of Mycobacterium is presented on Table 1. These strains were selected on the basis of previous studies that had revealed that most of these were sensitive to the test drugs including the phenothiazines at low concentrations. The MIC of INH, Py and $\mathrm{Sm}$ in the recent clinical isolates M.tuberculosis Bajaj 1, J15 and N23 was rather high. In M.tuberculosis Bajaj 1, the MIC of INH and Sm was $25 \mu \mathrm{g} / \mathrm{ml}$. The strain N23 was even more resistant, the MIC of Py and $\mathrm{Sm}$ was as high as $50 \mu \mathrm{g} / \mathrm{ml}$. With respect to the phenothiazines, the MIC values ranged between 10 and $25 \mu \mathrm{g} / \mathrm{ml}$ in most of the test strains; however, in case of Bajaj 1, J15 and N23 the MIC of Md and Pz was $50 \mu \mathrm{g} / \mathrm{ml}$.

Table 1 Minimum inhibitory concentration (MIC) of anti-tubercular drugs, methdilazine and promethazine against various strains of Mycobacterium

\begin{tabular}{|c|c|c|c|c|c|c|c|}
\hline \multirow{2}{*}{ Mycobacterium } & \multicolumn{7}{|c|}{ MIC $(\mu \mathrm{g} / \mathrm{ml})$} \\
\hline & INH & $\mathrm{Rf}$ & $\mathrm{Eb}$ & Py & $\mathrm{Sm}$ & Md & $\mathrm{Pz}$ \\
\hline M.smegmatis 789 & 2 & 2 & 2 & 5 & 2 & 25 & 10 \\
\hline M.smegmatis 1546 & 2 & 2 & 2 & 5 & 2 & 10 & 25 \\
\hline M.fortuitum 1529 & 2 & 2 & 2 & 2 & 5 & 10 & 25 \\
\hline M.scrofulaceum 1323 & 2 & 1 & 2 & 2 & 2 & 10 & 10 \\
\hline M.avium 724 & 5 & 5 & 10 & 2 & 10 & 25 & 25 \\
\hline M.gordonae 1324 & 2 & 2 & 2 & 5 & 2 & 10 & 10 \\
\hline M.phlei L1 & 5 & 2 & 2 & 10 & 2 & 25 & 25 \\
\hline M.marinum 50 & 2 & 2 & 5 & 5 & 2 & 25 & 25 \\
\hline M.intracellulare 1406 & 5 & 5 & 5 & 5 & 2 & 10 & 25 \\
\hline M.flavescens 1541 & 2 & 2 & 5 & 5 & 2 & 10 & 10 \\
\hline M.terrae 1450 & 5 & 2 & 2 & 5 & 2 & 10 & 10 \\
\hline M.tuberculosis $\mathrm{H}_{37} \mathrm{Ra} 16$ & 2 & 2 & 2 & 5 & 2 & 5 & 25 \\
\hline M.tuberculosis $\mathrm{H}_{37} \mathrm{Rv} 102$ & 2 & 2 & 5 & 5 & 2 & 10 & 25 \\
\hline M.tuberculosis Bajaj1 & 25 & 5 & 5 & 5 & 25 & 50 & 50 \\
\hline M.tuberculosis $\mathrm{J} 15$ & 25 & 5 & 10 & 25 & 25 & 50 & 50 \\
\hline M.tuberculosis $\mathrm{N} 23$ & 25 & 5 & 5 & 50 & 50 & 50 & 50 \\
\hline M.tuberculosis K1 & 2 & 1 & 2 & 5 & 2 & 10 & 25 \\
\hline M.tuberculosis 2 & 2 & 1 & 5 & 2 & 2 & 10 & 25 \\
\hline ICRC bacillus & 2 & 2 & 2 & 5 & 2 & 10 & 10 \\
\hline "Skinsness" bacillus & 2 & 2 & 5 & 5 & 2 & 10 & 25 \\
\hline
\end{tabular}

INH, isoniazid acid hydrazide; Rf, rifampicin; Eb, ethambutol; Py, pyrazinamide; Sm, streptomycin; Md, methdilazine; Pz, promethazine

\section{Selection of mutants}

All the 20 strains of Mycobacterium spp. were investigated for production of highly resistant Md mutants; of these 11 failed to produce mutants even after repeated tests. Nine strains of mycobacteria passaged on low but step-wise gradually rising concentrations of Md finally produced mutants of high resistance. The mutants were accepted only when the identification tests particularly with respect to cultural morphology in LJM coupled with acid fastness, established that they belonged to the respective wild-types. Following the same principle Pz mutants were selected. It may be pointed out here that $M$. tuberculosis Bajaj 1, J15, N23, $\mathrm{H}_{37} \mathrm{Rv} 102$ and $\mathrm{H}_{37} \mathrm{Ra} 16$ were able to develop fairly resistant mutants with respect to both $\mathrm{Md}$ and Pz. Among the others M.phlei L1, M.flavescens 1541, M.avium 724 and M.gordonae 1324 were able to produce Md- resistant mutants while 4 other strains of Mycobacterium could successfully develop Pz resistant mutants. The remaining 7 strains were unable to select drug resistant mutants. 


\section{Antibiotic cross-resistance patterns of Md and Pz-resistant mutants}

It may be noted from Table 2 that in $M$ phlei L1 the MIC of Md was $25 \mu \mathrm{g} / \mathrm{ml}$, the first step mutant could be developed at $50 \mu \mathrm{g} / \mathrm{ml}$ of $\mathrm{Md}$ when the MIC of Md was found to be $100 \mu \mathrm{g} / \mathrm{ml}$. From this the step 2 mutant of the same organism was developed whose MIC value of Md was $400 \mu \mathrm{g} / \mathrm{ml}$. There was a gradual increase in the MIC values with respect to $\mathrm{INH}, \mathrm{Rf}, \mathrm{Eb}$ and Py while there was no change in MIC values with respect to Sm. A similar pattern was noted in M.flavescens 1541. However, in the Md- mutants of M.avium 724 and
M.gordonae 1324, MIC values decreased in case of Sm and Py respectively. $M$. tuberculosis $\mathrm{H}_{37} \mathrm{Rv} 102$ and $\mathrm{Ra} 16$ being rather sensitive to test drugs, the levels of Md resistances in mutants of these strains were not so high and the changes in resistogramme pattern of mutants were not significant (Table 2). On the contrary, M. tuberculosis Bajaj 1, J15 and N23 being much less sensitive to the test agents, produced mutants at much higher levels of Md and simultaneously exhibited greater MICs with respect to all the antitubercular drugs except the strain N23 whose MIC value of Eb decreased in the mutants.

Table 2 Changing pattern of resistances of antimycobacterial drugs in methdilazine resistant mutants of Mycobacterium spp.

\begin{tabular}{|c|c|c|c|c|c|c|c|}
\hline \multirow[t]{2}{*}{ Mycobacteria } & \multirow[t]{2}{*}{ Types of cultures } & \multicolumn{6}{|c|}{$\begin{array}{l}\text { MIC }(\mu \mathrm{g} / \mathrm{ml} \text { in LJM) of different agents for the wild type and } \\
\text { mutant bacteria }\end{array}$} \\
\hline & & INH & Rf & $\mathrm{Eb}$ & $\mathrm{Py}$ & $\mathrm{Sm}$ & Md \\
\hline \multirow{4}{*}{ M.phlei L1 } & Wild Type & 5 & 2 & 2 & 10 & 2 & 25 \\
\hline & Md mutant step $1(50)$ & 5 & 2 & 2 & 10 & 2 & 100 \\
\hline & Md mutant step 2 (200) & 10 & 10 & 5 & 25 & 2 & 400 \\
\hline & Wild Type & 2 & 2 & 5 & 5 & 2 & 10 \\
\hline \multirow{3}{*}{ M.flavescens 1541} & Md mutant step 1 (25) & 5 & 2 & 5 & 25 & 10 & 100 \\
\hline & Md mutant step 2(200) & 5 & 2 & 10 & 25 & 25 & 500 \\
\hline & Wild Type & 5 & 5 & 10 & 2 & 10 & 25 \\
\hline \multirow{3}{*}{ M.avium 724} & Md mutant step $1(50)$ & 5 & 10 & 25 & 5 & 10 & 200 \\
\hline & Md mutant step 2(400) & 5 & 10 & 25 & 5 & 5 & 100 \\
\hline & Wild Type & 2 & 2 & 2 & 5 & 2 & 10 \\
\hline \multirow{3}{*}{ M.gordonae 1324} & Md mutant step $1(50)$ & 2 & 2 & 2 & 2 & 2 & 200 \\
\hline & Md mutant step 2(400) & 5 & 2 & 5 & 2 & 5 & 1000 \\
\hline & Wild Type & 25 & 5 & 5 & 5 & 25 & 50 \\
\hline \multirow{3}{*}{ M.tuberculosis Bajaj1 } & Md mutant step 1 (100) & 25 & 5 & 10 & 10 & 25 & 200 \\
\hline & Md mutant step 2(400) & 50 & 5 & 10 & 25 & 25 & 500 \\
\hline & Wild Type & 25 & 5 & 10 & 25 & 25 & 50 \\
\hline \multirow{3}{*}{ M.tuberculosis J15 } & Md mutant step 1 (200) & 25 & 5 & 25 & 10 & 25 & 500 \\
\hline & Md mutant step 2(1000) & 50 & 5 & 25 & 10 & 25 & 2000 \\
\hline & Wild Type & 25 & 5 & 5 & 50 & 50 & 50 \\
\hline \multirow{2}{*}{ M.tuberculosis N23 } & Md mutant step 1 (200) & 50 & 5 & 2 & 50 & 50 & 500 \\
\hline & Md mutant step 2(1000) & 100 & 5 & 2 & 100 & 50 & 2000 \\
\hline \multirow{3}{*}{$\begin{array}{l}\text { M.tuberculosis } \\
\mathrm{H}_{37} \mathrm{Rv} 102\end{array}$} & Wild Type & 2 & 2 & 5 & 5 & 2 & 10 \\
\hline & Md mutant step 1 (25) & 2 & 2 & 10 & 5 & 5 & 100 \\
\hline & Md mutant step 2(200) & 2 & 2 & 10 & 25 & 5 & 500 \\
\hline \multirow{3}{*}{ M.tuberculosis $\mathrm{H}_{37} \mathrm{Ra} 16$} & Wild Type & 2 & 2 & 2 & 5 & 2 & 5 \\
\hline & Md mutant step 1 (25) & 2 & 5 & 2 & 5 & 5 & 50 \\
\hline & Md mutant step 2(100) & 5 & 5 & 2 & 2 & 5 & 200 \\
\hline
\end{tabular}

*INH, isoniazid acid hydrazide; Rf, rifampicin; Eb, ethambutol; Py, pyrazinamide; Sm, streptomycin; Md, methdilazine

The step-wise mutants produced by mycobacteria against $\mathrm{Pz}$ showed nearly similar pattern of increase in resistances against the antitubercular drugs.
However, in case of M.intracellulare 1406 there was a loss in the MIC value of Eb (Table 3).

Table 3 Promethazine $(\mathrm{Pz})$ resistant mutants of Mycobacterium spp. and their effects on change of resistant patterns with respect to antimycobacterial drugs

\begin{tabular}{|c|c|c|c|c|c|c|c|}
\hline \multirow{2}{*}{ Mycobacteria } & \multirow{2}{*}{ Types of cultures } & \multicolumn{6}{|c|}{$\begin{array}{l}\text { MIC ( } \mu \mathrm{g} / \mathrm{ml} \text { in LJM) of different agents with respect to wild } \\
\text { type and mutant bacteria }\end{array}$} \\
\hline & & INH & $\mathrm{Rf}$ & $\mathrm{Eb}$ & Py & $\mathrm{Sm}$ & $\mathrm{Pz}$ \\
\hline \multirow{4}{*}{ M.fortuitum 1529} & Wild Type & 2 & 2 & 2 & 2 & 5 & 25 \\
\hline & Pz mutant step 1 (50) & 5 & 2 & 2 & 5 & 5 & 100 \\
\hline & Pz mutant step $2(200)$ & 5 & 2 & 5 & 25 & 10 & 400 \\
\hline & Wild Type & 2 & 1 & 2 & 2 & 2 & 10 \\
\hline \multirow{3}{*}{ M.scrofulaceum 1323} & Pz mutant step 1 (25) & 5 & 1 & 2 & 25 & 10 & 50 \\
\hline & Pz mutant step 2(100) & 5 & 2 & 10 & 25 & 25 & 400 \\
\hline & Wild Type & 2 & 2 & 5 & 5 & 2 & 25 \\
\hline \multirow{3}{*}{ M.marinum 50} & Pz mutant step $1(50)$ & 5 & 5 & 25 & 5 & 5 & 100 \\
\hline & Pz mutant step 2(200) & 5 & 10 & 25 & 5 & 5 & 400 \\
\hline & Pz mutant step 3(500) & 10 & 25 & 50 & 10 & 5 & 1000 \\
\hline \multirow{5}{*}{ M.intracellulare 1406} & Wild Type & 5 & 5 & 5 & 5 & 2 & 25 \\
\hline & Pz mutant step $1(50)$ & 5 & 2 & 5 & 5 & 2 & 200 \\
\hline & Pz mutant step 2(400) & 5 & 2 & 10 & 10 & 5 & 500 \\
\hline & Pz mutant step 3(1000) & 25 & 2 & 50 & 25 & 10 & 2000 \\
\hline & Wild Type & 25 & 5 & 5 & 5 & 25 & 50 \\
\hline \multirow{2}{*}{ M.tuberculosis Bajaj1 } & Pz mutant step $1(100)$ & 50 & 5 & 5 & 10 & 25 & 200 \\
\hline & Pz mutant step 2(400) & 100 & 5 & 10 & 25 & 50 & 1000 \\
\hline \multirow{4}{*}{ M.tuberculosis J15 } & Wild Type & 25 & 5 & 10 & 25 & 25 & 50 \\
\hline & Pz mutant step $1(100)$ & 25 & 10 & 25 & 10 & 50 & 200 \\
\hline & Pz mutant step 2(400) & 25 & 10 & 50 & 10 & 50 & 1000 \\
\hline & Wild Type & 25 & 5 & 5 & 50 & 50 & 50 \\
\hline \multirow{2}{*}{ M.tuberculosis N23 } & Pz mutant step $1(100)$ & 25 & 5 & 5 & 50 & 100 & 400 \\
\hline & Pz mutant step 2(500) & 100 & 5 & 10 & 100 & 100 & 1000 \\
\hline M.tuberculosis & Wild Type & 2 & 2 & 5 & 5 & 2 & 10 \\
\hline
\end{tabular}




\begin{tabular}{|c|c|c|c|c|c|c|c|}
\hline \multirow[t]{2}{*}{$\mathrm{H}_{37} \mathrm{Rv} 102$} & Pz mutant step 1 (25) & 5 & 2 & 5 & 10 & 2 & 50 \\
\hline & Pz mutant step 2(100) & 5 & 2 & 10 & 25 & 10 & 400 \\
\hline \multirow{3}{*}{ M.tuberculosis $\mathrm{H}_{37} \mathrm{Ra} 16$} & Wild Type & 2 & 2 & 2 & 5 & 2 & 5 \\
\hline & Pz mutant step 1 (10) & 2 & 2 & 2 & 5 & 2 & 25 \\
\hline & Pz mutant step 2(50) & 10 & 5 & 5 & 5 & 5 & 100 \\
\hline
\end{tabular}

*INH, isoniazid acid hydrazide; Rf, rifampicin; Eb, ethambutol; Py, pyrazinamide; Sm, streptomycin; Pz, promethazine

\section{DICUSSION}

Prevalence of cross-resistances between aminoglycosides in drug-resistant mutants of M.tuberculosis has been studied extensively. Resistance to kanamycin in viomycin-resistant strains and resistance to streptomycin in kanamycinresistant strains were two to four times greater than the resistant levels of the parent strains of M.tuberculosis (Torii et al., 1959; Steenken et al., 1959) Tsukamura (1974) isolated two types of tuberactinomycin $\mathrm{N}$ resistant mutants of M.tuberculosis, the first one was resistant to low levels of tuberactinomycin $\mathrm{N}$, viomycin and capreomycin, while the other was resistant at high levels to all these three antibiotics plus kanamycin and lividomycin. With the help of an intensive study Tsukamura and Mizuno in $\mathbf{1 9 7 5}$ proved that aminoglycoside antibiotics could be classified into three major types: streptomycin resistance, combined viomycin-tuberactinomycin $\mathrm{N}$ - capreomycin resistance, and the third type included resistance to kanamycin, lividomycin and paramomycin. No crossresistance between streptomycin and any other amionoglycoside antibiotic was observed. Although there is a large number of studies on clinical isolates of M.tuberculosis on mutations of genes and their relatedness of resistances to specific antibiotics (Taniguchi et al., 1996; Telenti et al., 1993; Ginsburg, 2005; Pitaksajjakul et al., 2005) evaluation of drug-resistance in experimentally produced mutants of M.tuberculosis has not been reported during past several years.

Imperiale et al (2014) studied cross-resistances to isoniazid, rifampicin and levofloxacin at a molecular level in clinical isolates of M.tuberculosis. With the help of microplate colorimetric method they determined MIC of isoniazid, ethionamide, rifampicin, rifabutin and moxifloxacin in the clinical isolates. Mutations conferring drug resistances were detected by GenoType MTBDR plus and DNA sequences. Isoniazid and ethionamide cross resistance was detected in $95.12 \%$ of isoniazid resistant isolates harbouring a mutation in inhAP or inhA open reading frame, but rifabutin cross-resistance was observed in $90 \%$ of clinical isolates originally shown to be resistant to rifampicin. This study highlighted that the same mutation causing resistance to the first line antitubercular drugs can be responsible for resistance to their respective structural analogs. Such findings are expected to help clinicians to decide on the treatment regime.

In this study it has been observed that Md mutants of M.phlei L1 developed cross-resistance to INH, Rf, $\mathrm{Eb}$ and $\mathrm{Py}$ in stepwise manner but not to $\mathrm{Sm}$; with respect to M.flavescens 1541 the Md mutants developed cross-resistances to INH, $\mathrm{Eb}, \mathrm{Py}, \mathrm{Sm}$ and not To Rf. Wild type clinical isolates M.tuberculosis Bajaj 1, J15 and N23 had much higher level of MIC of all the test drugs, except Rf. Surprisingly, step-up mutants of all these 3 strains failed to develop resistance to $\mathrm{Rf}$ as well. On the contrary, resistance level of Eb decreased in step-two mutants of M.tuberculosis N23. Similar decrease in MIC of Sm was noted in M.avium 724 (Table 2). The Pz-resistant step-three mutants of M.marinum 50 and M.intracellulare 1406 were resistant to $\mathrm{Pz}$ at very high levels and increase in MIC values of $\mathrm{Eb}$ in these mutants was also significantly high. The resistances of wild-type and mutant mycobacteria differed in most instances by sufficiently large margins (within the limits of confidence of the test system), and therefore appeared to be truly reflective of the actual resistance of such mycobacteria. It is unlikely that the low levels of resistances exhibited by some of the mutants were due to their reduced growth rate, incubation period was kept for sufficiently long period even for slow-growers and these resistances did not seem to depend on drug-modifying enzymes; these, therefore, should not affect their MIC determination. Indeed MICs of several test drugs were found to be elevated simultaneously. Thus these observations on cross-resistance seem to be more easily explainable on the basis of a non-specific reduction in cell membrane permeability of the various $\mathrm{Md}$ and $\mathrm{Pz}$ mutants in varying degrees and selectivity with respects to different test drugs. A literature survey on the MICs of different antibiotics shows that there had been a significant rise in the highest values that characterize drug resistant strains as well as the lowest values of MICs of drugs/antibiotics (characterizing sensitive strains) with respect to almost all groups of pathogenic bacteria during the last five decades since the beginning of antibiotic era (Garrod and O' Grady, 1971; Ray et al., 1980). Development of such resistances has been ascribed to "intrinsic resistance" which is an evolutionary ancient phenotype and can be defined as resistance of any bacterial species to a particular drug/antibiotic that has not been acquired as a result of exposure to such agents (Fajardo et al., 2008). Intrinsic resistance is the result of reduced permeability of bacterial envelope and the activity of efflux pumps (Nikaido and Zgurskaya, 1999). This suggests that the main physiological role of the components of intrinsic resistance involves the prevention of toxic components by restricting the permeability of the cell or the active export of toxic compounds. However, intrinsic resistance of M.tuberculosis has been traditionally attributed to the unusual structure of its mycolic acid containing cell wall that contributes to the low permeability for antitubercular antibiotics and chemotherapeutics (Jarker and Nikaido, 1994). The role of efflux mechanisms has been recognized as an important factor in the natural resistances of mycobacteria against tetracyclines, aminoglycosides and fluoroquinolones (De Rossi et al., 2006). Even though mutations in several genes are evidently related to drug resistance in M.tuberculosis a large number of clinical isolates do not seem to present such classical mutations (Almeida Da Silva and Palomino, 2011). It is known that inadvertent and extensive use of antitubercular drugs in tuberculosis patients have continuously contributed to substantial increase in drug resistances in M.tuberculosis resulting in emergence of multi-drug resistant and extensively drug resistant strains.

In view of our data and other findings it may be stated that the overall elevation of MIC of antitubercular drugs could also be due to a prolonged and massive use of a vast number chemotherapeutics, many of which have undetected or unsuspected antimicrobial/antitubercular activities. A few among such drugs are several phenothiazines including Md and Pz (Molnar et al., 1977; Kristiansen and Vergemann, 1986; Ratnakar and Murthy, 1993; Chakrabarty et al., 1993; Dutta et al., 2009; Crowle et al., 1992; Amaral et al.,1996; van Ingen et al., 2009; Advani et al., 2012; Kristiansen et al., 2015). It may be plausible to presume that many or all of these drugs could have selected mutants resistant to themselves and non-specifically to other antitubercular drugs all at low levels, at the therapeutic dosages at which they are used, thus contributing towards a gradual rise of the baseline of MIC of antitubercular agents. The present study provides experimental evidences for one of such possibilities.

\section{CONCLUSION}

The accelerated speed with which tuberculosis has spread in all communities throughout the world and the higher frequency of infection by multidrug and extensively drug resistant $M$. tuberculosis have resulted in an alarming situation. In this study it was observed that many strains of M.tuberculosis could be inhibited by both the phenothiazines methdilazine and promethazine.Thus these findings open up a new arena of treatment for multidrug and extensively drug resistant mycobacteria. It may be pointed out here that a structurally similar phenothiazine, thioridazine, has been repeatedly proved to be a potent antitubercular drug. Both the two compounds methdilazine and promethazine are routinely used as antihistaminic drugs while thioridazine is an effective antipsychotic drug. It needs to be pointed out here that since both methdilazine and promethazine are given to patients as antihistaminics and not as neuroleptics development of track resistance is not possible on the basis of prescription. Since the structurally similar neuroleptic drug thioridazine has repeatedly shown to be active against M.tuberculosis all such phenothiazines may be grouped together to define the results as a "class mechanism". When the antihistaminic compounds methdilazine and promethazine were allowed to produce mutants resistant to either of them at higher levels, the organisms showed elevated levels of MIC of tested antitubercular drugs. Such a phenomenon may be attributed as intrinsic resistance, which is the result of reduced permeability of bacterial envelope alongwith action of efflux pumps,which, in turn, result in prevention of toxic components into the cell. Therefore, it may be plausible that prolonged and indiscriminate use of such phenothiazines having antitubercular action, often unknowingly, may have allowed development of mutants resistant to themselves and simultaneously cross-resistances to many routinely used antitubercular drugs. 


\section{REFERENCES}

Advani, M.J., Siddique, I., Sharma, P. \& Reddy, H. (2012). Activity of trifluoperazine against replicating, Non-replicating and drug resistant $M$ tuberculosis. PLoS ONE, 7, 1-9. http://dx.doi.org/10.1371/journal.pone.0044245 Almeida Da Silva, P. E. \& Palomino, J. C. (2011). Molecular basis and mechanisms of drug resistance in Mycobacterium tuberculosis: classical and new drugs. Journal of Antimicrobial Chemotherapy, 66,14171430. http://dx.doi.org/10.1093/jac/dkr173

Amaral, L., Kristiansen, J.E., Abebe, L.S. \& Millett, W. (1996). Inhibition of the respiration of multi-drug resistant clinical isolates of Mycobacterium tuberculosis by thioridazine: potential use for initial therapy of freshly diagnosed tuberculosis. Journal of Antimicrobial Chemotherapy, 38, 1049-1053. http://dx.doi.org/10.1093/jac/38.6.1049

Barrow, G.I. \& Feltham, R.K.A. (2003). In : Cowan and steel's manual for the identification of medical bacteria, third ed. Cambridge University Press, Cambridge

Chakrabarty, A.N., Bhattacharya, C.P. \& Dastidar, S.G. (1993) Antimycobacterial activity of methdilazine(Md), an antimicrobic phenothiazine Acta Pathologica Microbiologica et Immunologica Scandinavica ,101, 449-454. http://dx.doi.org/10.1111/j.1699-0463.1993.tb00133.x

Crowle, J.A., Douvas, G.S. \& May, M.H. (1992). Chlorpromazine: A drug potentially useful for treating mycobacterial infections. Chemotherapy, 38, 410419. http://dx.doi.org/10.1159/000239036

Dastidar, S.G., Chaudhury, A., Annadurai, S., Roy, S., Mookerjee, M. \& Chakrabarty, A.N. (1995). In vitro and in vivo antimicrobial action of fluphenazine. Journal of Chemotherapy, 7, 201-206. http://dx.doi.org/10.1179/joc.1995.7.3.201

Dastidar, S.G., Debnath, S., Mazumdar, K., Ganguly, K. \& Chakrabarty, A.N (2004). Triflupromazine: a microbicide non-antibiotic compound. Acta $\begin{array}{lll}\text { Microbioliogica et Immunologica Hungary, 51,75-83 } & \end{array}$ http://dx.doi.org/10.1556/amicr.51.2004.1-2.5

Dastidar, S.G., Kristiansen, J.E., Molnar, J. \& Amaral, L. (2013). Role of phenothiazines and structurally similar compounds of plant origin in the fight against infections by drug resistant bacteria. Antibiotics, 2, 58-71. http://dx.doi.org/10.3390/antibiotics2010058

De Rossi, E., Ainso, J.A. \& Riccardi, G. (2006). Role of mycobacterial efflux transporters in drug resistance : an unresolved question. FEMS Microbiology Review, 30, 36-52. http://dx.doi.org/10.1111/j.1574-6976.2005.00002.x

Dutta, N.K., Mazumdar, K., Dasgupta, A. \& Dastidar, S.G. (2009). Activity of the phenothiazine methdilazine alone or in combination with isoniazid or streptomycin against Mycobacterium tuberculosis in mice. Journal of Medical Microbiology, 58, 1667-1668. http://dx.doi.org/10.1099/jmm.0.014027-0

Fajardo, A., Martinez-Martin, N., Mercadillo, M., Galtan-galan, J.C., Ghysels, B., Matthijs, S., Cornelis, P., Weihlmann, L. , Tummler, B., Baquerro, F. \& Martinez, J.L. (2008). The neglected intrinsic resistome of bacterial pathogens. PLoS ONE, 3, e1619. http://dx.doi.org/10.1371/journal.pone.0001619

Garrod, L.P. \& O’Grady, F. (1971). Antibiotics \& Chemotherapy. E. \& S. Livingstone, Edinburgh \& London.

Ginsburg, A. (2005). Emergence of fluoroquinolone resistance in Mycobacterium tuberculosis during continuously dosed moxifloxacin monotherapy in a mouse model. Antimicrobial Agents and Chemotherapy, 49,3977-3979. http://dx.doi.org/10.1128/aac.49.9.3977-3979.2005

Imperiale, B., Di Giulio, A.B., Cataldi, A.A. \& Morcillo, N.S. (2014). Evaluation of Mycobacterium tuberculosis cross-resistance to isoniazid, rifampicin an levofloxacin with their respective structural analogs. The Journal of Antibiotics, 67,749-754

http://dx.doi.org/10.1038/ja.2014.61

Jarker, V. \& Nikaido, H. (1994). Mycobacterial cell wall structure and role in natural resistance to antibiotics. FEMS Microbiology Letters, 123, 11-18 http://dx.doi.org/10.1111/j.1574-6968.1994.tb07194.x

Kamerbeek, J., Schouls, L., Kolk, A., van Agterveld, M., van Soolingan, D., S. Kujiper, et al. (1997). Simultanoeus detection and strain differentiation of Mycobacterium tuberculosis for diagnosis and epidemiology. Journal of Clinical Microbiology, 35, 907-914.

Koseki, Y. \& Okamoto, S. (1963). Studies on cross-resistance between capreomycin and certain other anti-mycobacterial agents. Japanese Journal of Medicinal Science and Biology,16,

$1-38$

http://dx.doi.org/10.7883/yoken1952.16.31

Kristiansen, J.E. (1979). Experiments to illustrate the effect of chlorpromazine on the permeability of bacterial cell wall. Acta Pathologica Microbiologica et Immunologica Scandinavica, 87, 317-319. http://dx.doi.org/10.1111/j.16990463.1979.tb02445.x

Kristiansen, J.E. \& Vergmann, B. (1986). The antibacterial effect of selected phenothiazines and thioxanthenes on slow growing mycobacteria. Acta Pathologica Microbiologica et Immunologica Scandinavica, 94, 393-398. http://dx.doi.org/10.1111/j.1699-0463.1986.tb03073.x

Kristiansen, J.E., Dastidar, S.G., Palchoudhuri, S., Sinha Roy, D., Das, S. Hendricks, O. \& Christensen, J.B. (2015). Phenothiazones as a solution for multidrug resistant tuberculosis: from the origin to present. International Microbiology, 18, 1-12
Mazumdar, R., Ganguly, K., Dastidar, S.G. \& Chakrabarty, A.N. (2001) Trifluoperazine: A broad-spectrum bactericide specially active on staphylococci and vibrios. International Journal of Antimicrobial Agents, 18, 403 406. http://dx.doi.org/10.1016/s0924-8579(01)00324-7

Maus, C.E., Plikaytis, B.B. \& Shinnick, T.M. (2005). Molecular analysis of cross-resistance to capreomycin, kanamycin, amikacin, and viomycin in Mycobacterium tuberculosis. Antimicrobial Agents and Chemotherapy, 49, 3192-3197. http://dx.doi.org/10.1128/aac.49.8.3192-3197.2005

Molnar, J., Beladi, I. \& Foldes, I. (1977). Studies on anti-tuberculotic action of some phenothiazine derivatives in vitro. Zentralblatt für Bakteriologie (Orig A), 239, 521-526

Nikaido, H. \& Zgurskaya, H.I. (1999). Antibiotic efflux mechanisms. Current Opinion on Infectious Diseases, 12,529-36. http://dx.doi.org/10.1097/00001432199912000-00001

Pitaksajjakul, P., et al. (2005). Mutations in the gyrA and gyrB genes of fluoroquinoloneresistant Mycobacterium tuberculosis from TB patients in Thailand, Southeast Asian Journal of Tropical Medicine and Public Health, 36, 228-237.

Radhakrishnan, V., Ganguly, K., Ganguly, M., Dastidar, S.G. \& Chakrabarty, A.N. (1999). Potentiality of tricyclic compound thioridazine as an effective antibacterial and antiplasmid agent. Indian Journal of Experimental Biology, 37 671-675.

Ratnakar, P. \& Murthy, P.S. (1993). Trifluoperazine inhibits the incorporation of labelled precursors into lipids, proteins and DNA of Mycobacterium tuberculosis H37Rv. FEMS Microbiology letters, 110, 291-294 http://dx.doi.org/10.1016/0378-1097(93)90117-k

Ray, S., Dastidar, S.G. \& Chakrabarty, A.N. (1980). Antibiotic cross-resistance patterns of ambodryl and promazine resistant mutants. British Journal of Experimental Pathology, 61,465.

Steenken, W., Montalbine, V. \& Thurstonj, R. (1959). The antituberculous activity of kanamycin in vitro and in the experimental animal (guinea pig) American Review of Tuberculosis, 79 , 66-71. http://dx.doi.org/10.1111/j.17496632.1958.tb54696.x

Suzuki, Y., Katsukawa, C., Tamaru, A., Abe, C., Makino, M., Mizuguchi, Y. \& Taniguchi, H. (1998). Detection of kanamycin-resistant Mycobacterium tuberculosis by identifying mutations in the 16S rRNA gene. Journal of Clinical Microbiology, 36,1220-1225.

Taniguchi, H., Aramaki, H., Nikaido, Y., Mizuguchi, Y., Nakamura, M., Koga, T. \& Yoshida, S. (1996). Rifampicin resistance and mutation of the $r p o B$ gene in Mycobacterium tuberculosis. FEMS Microbiology Letters, 144,103-108. http://dx.doi.org/10.1111/j.1574-6968.1996.tb08515.x

Telenti, A., Imboden, P. , Marchesi, F., Lowrie, D., Cole, S., Colston, M.J. Matter, L., Schopfer, K. \& Bodmer, T. (1993). Detection of rifampicin-resistance mutations in Mycobacterium tuberculosis. Lancet, 341,647-650. http://dx.doi.org/10.1016/0140-6736(93)90417-f

Torii, F., Yamamoto, M., Hayashi, M., Noda, Y., \& Tsukamura, M. (1959) Studies on the kanamycin resistance of Mycobacterium tuberculosis. II Kanamycin-sensitivity of various drug-resistant strains. Journal of Antibiotics, 12 ,103-104.

Tsukamura, M. (1959). Further studies on the one-way cross-resistance in Mycobacterium tuberculosis with special reference to streptomycin resistance kanamycin resistance, and viomycin resistance. Japanese Journal of Genetics, 34, 275-28. http://dx.doi.org/10.1266/jijg.34.275

Tsukamura, M. (1969). Cross-resistance relationships between capreomycin, kanamycin and viomycin resistances in tubercle bacilli from patients. American Review of Respiratory Diseases, 99, 780-782.

Tsukamura , M. (1974). Resistance pattern to tuberactinomycin-N and cross resistance relationships between tuberactinomycin-N, lividomycin, capreomycin, viomycin, kanamycin and streptomycin resistances of Mycobacterium tuberculosis $\left(\mathrm{H}_{37} \mathrm{Rv}\right)$. Chemotherapy (Tokyo), 22, 1115-1121.

Tsukamura, M. \& Mizuno, S.(1975). Cross-resistance relationships among the aminoglucoside antibiotics in Mycobacterium tuberculosis. Journal of General Microbiology, 88, 263-274. http://dx.doi.org/10.1099/00221287-88-2-269

Van Ingen, J., van der Laan, T., Amaral, L., Dekhuijzen, R., Boeree, M.J. \& van Soolingen, D. (2009). In vitro activity of thioridazine against mycobacteria. International Journal of Antimicrobial Agents, 34,190-191. http://dx.doi.org/10.1016/j.ijantimicag.2009.02.015

World Health Organization (2013) Global Tuberculosis Report. WHO, Geneva, Switzerland 